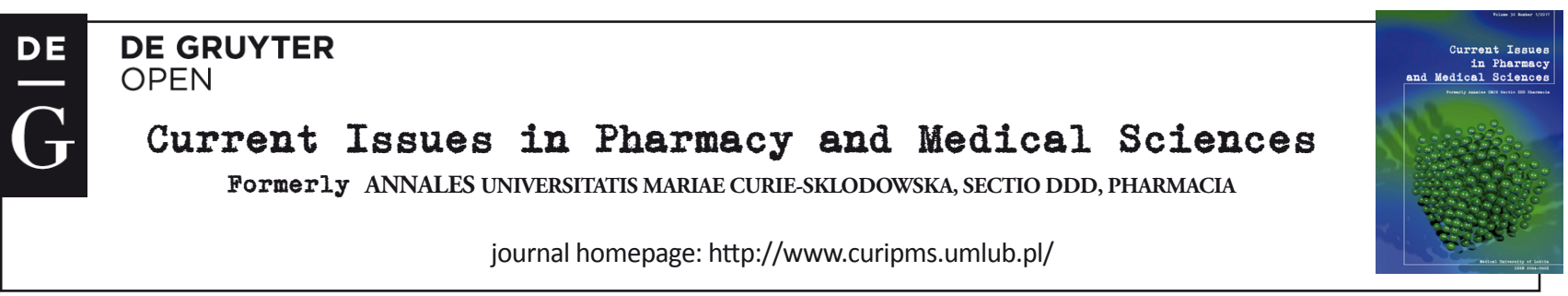

\title{
Treatment of peritoneal metastases from gastric carcinoma
}

\author{
Wojciech P. Polkowski, Karol Rawicz-Pruszynski, Jerzy Mielko, \\ Katarzyna Geca, Magdalena Skorzewska, Bogumila Cisel
}

Department of Surgical Oncology, Medical University of Lublin, Staszica 11, 20-081 Lublin, Poland

\begin{tabular}{|c|c|}
\hline ARTICLE INFO & ABSTRACT \\
\hline $\begin{array}{l}\text { Received } 22 \text { December } 2017 \\
\text { Accepted } 29 \text { December } 2017\end{array}$ & $\begin{array}{l}\text { Patients with advanced gastric cancer and positive peritoneal cytology and/or peritoneal } \\
\text { dissemination are deemed to be incurable and to hold dismal prognosis. So far, the }\end{array}$ \\
\hline $\begin{array}{l}\text { Keywords: } \\
\text { gastric cancer, } \\
\text { peritoneal metastases, } \\
\text { gastrectomy, } \\
\text { hyperthermic intraperitoneal } \\
\text { chemotherapy, } \\
\text { cytoreductive surgery. }\end{array}$ & $\begin{array}{l}\text { only treatment option for these patients has been palliative systemic (chemo)therapy. } \\
\text { However, for the last three decades, great progress has been made in attempts to treat } \\
\text { (potential) peritoneal dissemination by means of complete cytoreductive surgery } \\
\text { combined with hyperthermic intraperitoneal chemotherapy (HIPEC) after preoperative } \\
\text { systemic therapy. This review is focused on the recent achievements of this multimodal } \\
\text { strategy. Additionally, the review stands as background for the } 4^{\text {th }} \text { International } \\
\text { Conference "Advances in Surgical Oncology" that was held at the Medical University of } \\
\text { Lublin (Poland) in November 2017, and dedicated to cytoreductive surgery and HIPEC } \\
\text { for advanced gastric cancer. }\end{array}$ \\
\hline
\end{tabular}

\section{PERITONEAL METASTASES}

The peritoneum is the main site of gastric cancer (GC) metastases. Whereas haematogenous metastases to the liver and other distant organs are found in $40 \%$ of patients, these are present in 53-60\% of GC patients [1]. Indeed, nearly half of the patients suffering from advanced GC will develop peritoneal metastases (PM) due to stomach serosa involvement by the tumour [2]. Optimal and standardised treatment of PM from $\mathrm{GC}$ is yet to be defined.

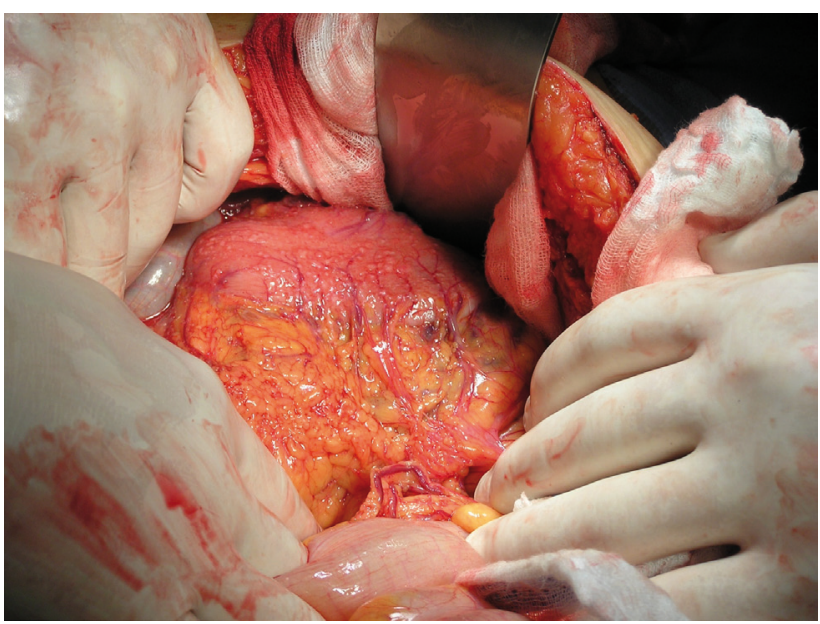

Figure 1. Extensive peritoneal metastases in a patient with gastric carcinoma at laparotomy

\footnotetext{
* Corresponding author

e-mail: wojciech.polkowski@uml.edu.pl
}

A randomized controlled GYMSSA trial compared gastrectomy with metastasectomy followed by systemic treatment versus systemic therapy alone in advanced GC [4]. This extensive surgical approach involved removing all cancer deposits, and it contrasts with the REGATTA trial design, in which patients underwent only limited (palliative, cytoreductive) surgery (D1 gastrectomy) without metastasectomy.

Until further evidence is shown, the REGATTA trial showed no survival benefit in patients which were treated surgically prior to chemotherapy [3]. Therefore, in advanced and non-curable GC (with PM), chemotherapy is recommended as a standard treatment. The limited permeability of the peritoneal plasma barrier allows the delivery of high concentrations of chemotherapeutic drugs directly into the peritoneal cavity, without the danger of high plasma concentrations and subsequent systemic toxicity.A lack of effective systemic therapy and the fact that locally advanced GC with PM is most likely considered as unresectable, has triggered surgical researches towards hyperthermic intraperitoneal chemotherapy (HIPEC) in neo- and adjuvant, as well as in palliative (in patients with ascites) and prophylactic (positive cytology in the peritoneal fluid sample) settings [5]. HIPEC now has an established role in the treatment of peritoneal carcinomatosis originating from colorectal cancer, including appendiceal cancer, mesothelioma, and pseudomyxoma peritonei. The greater understanding of the biopathology of peritoneal dissemination combined with peritonectomy 
(removal of macroscopic lesions) and HIPEC (elimination of microscopic disease) has allowed a therapeutical shift from palliative only, to radical application, with intention to treat in carefully selected group of patients.

The 30-year experience of the HIPEC in the treatment of advanced stage GC with and without PM, has been compared with standard oncological management in a metaanalysis of randomized and high quality non-randomized studies [6]. The comparison has demonstrated the survival advantage of the use of HIPEC as a prophylactic strategy and suggested that patients whose disease burden is limited to positive cytology and limited nodal involvement may benefit the most from HIPEC.

\section{DIAGNOSIS OF PERITONEAL METASTASES FROM GC}

Until recently, peritoneal dissemination was diagnosed during laparotomy with curative-intent gastric resection due to the low detection rate during pre-operative staging with computed tomography (CT). However, the introduction of diagnostic laparoscopy in patients lacking evident distant metastases in preoperative imaging tests has led to minimally invasive peritoneal cavity assessment prior to radical gastrectomy.

Further improvement of disease staging is available through direct visualisation of suspected malignancies on the peritoneal surface, the cytological assessment of peritoneal fluid (by way of the usage of peritoneal lavage in order to obtain free cancer cells) and through laparoscopic ultrasonography $[7,8]$. The lack of macroscopically visible PM, yet a positive cytological examination is considered effective assessment, and determines the metastatic disease - M1 $[9,10]$. Positive peritoneal cytology is most likely to occur in T3/T4 primary tumours and it is considered to be an important prognosticator and supplement of standard staging methods [11].

Except for early or advanced (M1) GC, staging laparoscopy should be performed prior to any clinical decisionmaking [12]. When planning an intention-to-treat resection, diagnostic laparoscopy is highly anticipated in the following setting:

- cT3/T4 tumour suspicion,

- multivisceral resection planning,

- when preoperative imaging suggests lymph node metastases $(\mathrm{N}+)$.

The staging of laparoscopy should include inspection of stomach, diaphragm, liver and ovaries in female patients, followed by Papanicolaou-staining cytological examination of the obtained peritoneal fluid [13].

\section{RESULTS OF CYTOREDUCTIVE SURGERY AND HIPEC}

The results of potential benefit from cytoreductive surgery (CRS) and HIPEC in 15 GC patients with PM from the first Japanese trial were published in 1988 [14]. Accordingly, the complications rate was considered to be acceptable and the overall survival (OS) was somewhat higher (7,2 \pm 4,6 months) than the current norm. Yonemura et al. then focused on the role of CRS and published the results of the surgical treatment of 41 patients that was followed by HIPEC perfusion with mitomycin $\mathrm{C}$ (MMC) and cisplatin [15]. Herein the median of OS was 14,6 months and 3-year OS estimated at $28,5 \%$. Furthermore, the long-term results of surgery combined with HIPEC with MMC in 48 patients showed 5-year and 8-year OS in, consecutively, 31 and 25\% of all patients [16]. Yonemura et al. also presented the results from one of the first major observational cohort studies [17]. In this, 5-year OS was reached in $13 \%$ of all patients after complete cytoreduction $(\mathrm{CC} 0 / 1)$, in comparison with $6 \%$ of all patients with incomplete $(\mathrm{CC} 2 / 3)$ cytoreduction.

Similarly to other peritoneal surface malignancies, in patients with advanced GC, the completeness of cytoreduction (CC) scale is used. The scale indicates complete cytoreduction (CC-0/1) and incomplete cytoreduction (CC $-2 / 3$ ), depending on the size of the peritoneal tumour that remained after cytoreduction. Thus, CC-0 indicates lack of residual disease; $\mathrm{CC}-1$ : the persisting nodules are smaller than $2.5 \mathrm{~mm}$; CC-2: such nodules are between $2.5 \mathrm{~mm}$ and $2.5 \mathrm{~cm}$, and CC-3: the nodules are greater than $2,5 \mathrm{~cm}$ [22]. Of note, complete cytoreduction (CC-1) is an equivalent to macroscopically non-radical resection $(\mathrm{R} 2 \mathrm{a})$.

The first multicentre retrospective study was performed in France among 159 patients [18]. herein, five-year OS was doubled after complete cytoreduction (23\%), in comparison with incomplete cytoreduction (13\%).

Most commonly, in clinical practice, $\mathrm{PM}$ in GC patients are diagnosed at an advanced stage of the disease, where resection and HIPEC are no longer possible. Thus, Yonemura proposed the application of so-called bidirectional chemotherapy - intraperitoneal and systemic, applied as a neo-adjuvant treatment (Neoadjuvant IntraPeritoneal and Systemic chemotherapy; NIPS) [19]. Its role is to downsize the primary tumour and eradicate free cancer cells along with engendering infiltrations of the peritoneal surface and subperitoneal vessels prior to CRS (gastrectomy) and HIPEC. The results of such a treatment in 194 GC patients with synchronous and metachronous PM were published in 2014 [20]. Accordingly, NIPS chemotherapy allowed the performance of CRS and HIPEC in 152 patients (73\%). Moreover, morbidity and mortality rates were $24 \%$ and $4 \%$, respectively. Finally, the median OS was 16 months, whereas 1-, 2- and 5- year OS were 66, 32 and 11\%, respectively.

Meta-analysis of 20 prospective studies, including 2145 advanced GC patients with PM treated with intraperitoneal chemotherapy has made it possible to consider the approach as an effective treatment after curative resection [21]. The meta-analysis showed that the odds ratio (OR) was 0,99 , with $95 \%$ confidence interval between 0,71 to 1,37 . What is more, the OS is improved when CRS is combined with intraperitoneal chemotherapy. On the contrary, it does not change the prognosis of patients with tumours with lymph node involvement or with serosa infiltration. Intraperitoneal chemotherapy, however, diminishes the risk of peritoneal recurrence and distant metastases. Still, it increases the risk of postoperative complications. Over all, HIPEC is not contraindicated in the presence of lymph node metastases. These studies show the advantages that arise from pre- and peri-operative intraperitoneal chemotherapy in GC patients 
with peritoneal dissemination., as NIPS combined with CRS and HIPEC increase the OS rate in these patients [21].

Despite the evidence from the afore-mentioned metaanalysis, an international expert working group reached a consensus on intraperitoneal chemotherapy [22]. It was recognised as inapplicable in GC patients with Peritoneal Carcinomatosis Index (PCI) greater than 20, in patients with peritoneal and synchronous metastases to both ovaries or other distant organs, as well as in patients who underwent multivisceral resection for T4 tumour without the presence of peritoneal dissemination [22].

So far only one prospective randomized controlled study has confirmed the efficacy of the CRS and HIPEC in the treatment of GC. Yang et al. published the results of a study with an experimental treatment arm that consisted of both CRS and HIPEC, whereas in the control group, only CRS was performed [23]. In the study, cytostatics (MMC and cisplatin) were administered intraperitoneally for 60 minutes at the temperature of $42^{\circ} \mathrm{C}$. Yang et al. noted that patients who underwent CRS and HIPEC had significantly longer OS, in comparison to the CRS only group. The authors, hence, concluded that HIPEC based on MMC and cisplatin might improve OS. In such studies, the most common cytostatics used for the HIPEC are MMC, docetaxel and cisplatin [24].

The meta-analysis of 8 randomized controlled trials validating the impact of HIPEC on OS after CRS showed significant improvement of 1-,2- and 3- year survival [21]. Additionally, HIPEC has a positive influence on the prevention of peritoneal recurrence after the curative surgery of serosa-infiltrating tumours. A multivariate analysis has revealed that the independent prognostic factors are: complete cytoreduction (CC0/1), PCI under cut-off value (of 12 points), histopathological response to neoadjuvant treatment and HIPEC [25].

Contrarily, according to the results of another study, HIPEC might increase the rate of postoperative complications, including the development of intra-abdominal abscesses and neutropenia (the presence of postoperative complications is considered to be an independent factor of poor prognosis) [26]. Still, meta-analysis did not show significant differences in postoperative mortality rate between HIPEC and control groups [27]. Therefore, HIPEC is considered to be safe and effective treatment, even following CRS. Currently, there are two on-going European phase III trials which are planned to determine the role of HIPEC in the surgical treatment of GC with PM [28,29].

For patients with extensive PM, the completeness of cytoreductive surgery is a critical prognostic factor for survival [6]. This was also shown in a previous meta-analysis [30]. Herein, one, 2, 3 and 5-year OS is increased by CC0-CC1 cytoreduction in patients with $\mathrm{PC}$ from gastric origin. What is more, $\mathrm{CC} 0$ increases the 1 and 3 years survival when compared to $\mathrm{CC} 1$ cytoreduction [30]. However, it is unlikely that the results of such application is a curative treatment. Still, CRS and HIPEC is associated with improved survival compared with gastrectomy or palliative chemotherapy alone, and patients treated with CRS and HIPEC have comparable survival to matched control patients without PM [31]. It must be underlined, however, that regardless of treatment strategy, nearly all patients subsequently experienced GC recurrence and died of the disease.

\section{CYTOSTATICS USED IN HIPEC}

Although among surgeons with intraperitoneal treatment expertise, it is generally accepted to combine extensive CRS (gastrectomy, peritonectomy and multivisceral resections) with HIPEC, there is still a lack of agreement for choice of intraperitoneally administered cytostatics or their combination. In the literature there is lack of evidence for the superiority of one group of chemotherapeutic agents over another in terms of outcomes, toxicity, side-effects and safety for medical staff in the operating room setting [32]. Most common cytostatic agents used intraperitoneally include MMC and cisplatin (or their compounds) in various dosage, temperatures and perfusion times.

In most institutions, HIPEC is performed deliberately using either an open or closed technique, with $\mathrm{MMC}$ in monotherapy $\left(10-40 \mathrm{mg} / \mathrm{m}^{2}\right.$ in $42^{\circ} \mathrm{C}$ for $60-90$ minutes) or in combination with cisplatin (CDDP). Docetaxel $\left(15 \mathrm{mg} / \mathrm{m}^{2}\right)$ is also frequently combined with CDDP $(75 \mathrm{mg} / \mathrm{m} 2$ in $41^{\circ}-42^{\circ} \mathrm{C}$ for 60 minutes). Intraperitoneal polychemotherapy regimens (MMC, CDDP with 5-FU or etoposide) are rarely used.

In the French randomized multicenter trial GASTRICHIP, monotherapy with oxaliplatin $\left(250 \mathrm{mg} / \mathrm{m}^{2}\right.$ with 2 liters of $5 \%$ glucose $/ \mathrm{m}^{2}$ at $42^{\circ}-43^{\circ} \mathrm{C}$ for 30 minutes) is constantly used [32]. One must note that the patients treated in the experimental arm of that trial receive intravenous 5-FU (400 $\mathrm{mg} / \mathrm{m}^{2}$ plus calcium levofolinate $10 \mathrm{mg} / \mathrm{m}^{2}$ ) as systemic chemotherapy induction for HIPEC 15 minutes before HIPEC begins.

Since currently there is no consensus on the choice of chemotherapeutic agents used in HIPEC for GC, a prerequisite for future HIPEC studies is to determine optimal regimen, as well as the maximum tolerated dose (MTD) of intraperitoneal drug combinations. Such a combination (docetaxel with a fixed dose of intraperitoneal oxaliplatin) is currently tested in the multicentre, open label, phase I-II dose-escalation PERISCOPE study [33].

\section{PREOPERATIVE LAPAROSCOPIC PCI EVALUATION}

The reliability of CT or positron emission tomography (PET) in staging and determining PCI is low, especially when the diameter of PM is below $10 \mathrm{~mm}[34,35]$. Particularly difficult for radiological assessment are small nodules located on the mesentery. Indeed, preoperative evaluation of PCI in GC patients by Hong et al. showed that PM were undetected in 39 (29\%) among 137 patients [36]. Currently, there is no adequate imaging test which would precisely determine the PCI.

Recently, diagnostic laparoscopy is being performed to accurately assess the PCI, as well to obtain histo- and cytological material from peritoneal surface and peritoneal fluid lavage, respectively. Preoperative laparoscopy is highly accurate in determining the PCI since its value is nearly identical when assessed during laparotomy [37]. If the peritoneal fluid was obtained before chemotherapy, the 
frequency of positive cytology in patients without apparent PM is estimated at 7-10\% [38,39].

A positive result of peritoneal fluid cytological examination, despite the lack of macroscopically visible peritoneal dissemination, allows to stage the disease as M1 according to the 7th edition of TNM Classification from 2010. In Poland, positive cytology is detected in $24 \%$ of all patients initially scheduled for radical surgery [40]. Factors that are predictive for positive cytology of peritoneal fluid include: stage T3/4, lymph node involvement, grade score G3, infiltration by the tumour of the entire stomach and indications of a diffuse type of GC by Laurén classification. The identification of patients in which diagnostic laparoscopy with lavage indicate peritoneal dissemination affects the clinical decision, arbitrary targeting them for the first line systemic therapy. Still, treatment of the signet-ring cell GC remains unresolved, but there are arguments for upfront surgery due to high risk of progression on induction/preoperative chemotherapy.

\section{PREVENTION OF PERITONEAL METASTASES}

Positive cytology of peritoneal fluid in GC patients is determined as "cy+" in the TNM classification of UICC. Japanese classification divides PM in GC into two subgroups: $\mathrm{P} 0$ and $\mathrm{P} 1$, similarly to the grouping of the cytology of peritoneal fluid: CY0 and CY1 [41]. Thusly, the following clinical settings are possible: $\mathrm{P} 0 / \mathrm{CY} 1$ reveals lack of macroscopically visible PM and positive cytology, whereas P1 indicates macroscopical dissemination of peritoneum +/- positive peritoneal cytology. Macroscopically radical gastrectomy performed with curative intent in CY1 patients is considered to be microscopically non-radical (R1).

The survival of $\mathrm{P} 0 / \mathrm{CY} 1$ patients classified as stage IV of GC are similar to P1 patients [41]. Such a situation clearly indicates that prognosis in GC patients with serosa infiltration and free cancer cells in the peritoneal cavity are comparable to patients with macroscopic PM. Recent data from the meta-analysis of randomized studies emphasize the advantage of intraperitoneal chemotherapy for preventive (prophylactic) treatment of advanced GC without macroscopic PM (P0) [21]. Furthermore, to achieve prolonged survival, performance of the CRS (most preferably CC-0) is essential, as shown in different meta-analysis [30]. Yet, the broadening of indication criteria for HIPEC use in all patients (even CY-/P0) with high risk of peritoneal dissemination (T4a; tumour invasion is contiguous to the serosa or penetrates the serosa and is exposed to the peritoneal cavity) remains a matter of debate, despite the solid validation. Infiltration of gastric serosa should, hence, be considered as a clinical situation in which the future development of PM is inevitable [42]. Of note, the frequency of peritoneal GC recurrence after curative resection increases with increased depth of invasion of the gastric wall (subserosal invasion, 35\%; serosal invasion, 47\%; and invasion of adjacent organs, $60 \%$ ) [43].

A review of the literature on the usefulness of HIPEC in the prevention of peritoneal recurrence after potentially radical surgery for GC was presented by Roviello et al. [27]. Herein, a number of phase III trials from the Far East, together with three meta-analyses confirm the beneficial effects of the HIPEC procedure. Such trials came about because the limitations of perioperative cytological diagnostics induced many Asian scientists to carry out research on HIPEC so as to supplement radical surgery in patients without macroscopic PM but with serosal invasion of the primary tumour. Three meta-analyses confirmed that such strategy, especially in combination with $\mathrm{R} 0$ resection, might be more effective than standard treatment in terms of minimizing peritoneal recurrence, without significant increase of postoperative complications [44-46].

Basically, HIPEC is used preventively as adjuvant treatment when PM are not macroscopically visible. Such treatment is based on peritoneal perfusion with cytostatic solutions in elevated $\left(42-43^{\circ} \mathrm{C}\right)$ temperatures or without heating the solution (ang. normotherpmic intraperitoneal chemotherapy; NIPEC) at the end of surgery, or in the early (early postoperative intraperitoneal chemotherapy; EPIC) or delayed (delayed postoperative intraperitoneal chemotherapy; DIPEC) postoperative period in GC patients with high risk of peritoneal recurrence. Based on meta-analysis data from 1648 patients, there was significant improvement of survival after HIPEC or EPIC [47]. A positive impact on survival was also observed after NIPEC, while there was no significant outcome after EPIC alone, nor after DIPEC. In almost every randomized trials from the Far East, HIPEC showed potential benefits in adjuvant treatment of patients with high risk of PM after radical surgery for advanced GC, especially in the diffuse or mixed Laurén type, in serosa infiltration $(\geq \mathrm{T} 4 \mathrm{a})$ or in positive cytology indications $(\mathrm{c}+/$ CY1) [27]. Unfortunately, standardization of the HIPEC procedure, especially in terms of cytostatic choice, is yet to be internationally confirmed [48].

The positive results from Far East should, hence, be confirmed in Western studies. Until now, in Europe, the available data has been limited to retrospective studies of minor groups comprising prophylactic HIPEC with surgical treatment only $[49,50]$.

\section{SYSTEMIC CHEMOTHERAPY - SURGERY - HIPEC SEQUENCE}

Unlike other peritoneal surface malignancies effectively treated by CRS and HIPEC, in GC, a PCI cut-off level for good prognosis is considered to be of only 12 points. All patients with PCI above the cut-off level, which is an independent prognostic factor, die of GC even after complete cytoreduction with HIPEC. Since even $70 \%$ of patients with PM show a PCI score above the cut-off level at the time of diagnosis, a crucial effort is to reduce intraperitoneal tumour burden and to eradicate peritoneal free cancer cells by an preoperative bidirectional treatment.

Yonemura et al., based on his large experience, put forward a comprehensive intraperitoneal treatment of GC with PM [51]. The suggested procedure consists of several consecutive steps to diminish the amount of residual cancer cells:

1. staging laparoscopy to diagnose PCI and histology, plus HIPEC 
2. neoadjuvant laparoscopic hyperthermic intraperitoneal chemoperfusion (NLHIPEC),

3. neoadjuvant intraperitoneal/systemic chemotherapy (NIPS) - 3 times,

4. re-staging laparoscopy plus HIPEC

5. followed by open surgery if regression is obtained

6. extensive intraperitoneal lavage (EIPL) before CRS,

7. CRS with peritonectomy,

8. EIPL after CRS,

9. HIPEC,

10. EPIC,

11. late postoperative systemic chemotherapy within 2 months.

In the proposal, diagnostic laparoscopy is performed in order to assess the peritoneal cancer index (PCI) and obtain confirmation of PM or the presence of free cancer cells in the peritoneal fluid as assessed in cytological and histological examination. An obvious limitation of CRS is high PCI and extensive carcinomatosis of small intestine and its mesentery. In order to lower PCI and to preserve the peritoneum itself, multiple (cyclic) LHIPEC and NIPS prior to CRS are generally performed. In standard practice, after the laparoscopic staging of the disease, EIPL (sodium chloride solution is administered through HIPEC device) is carried out in order to wash out and eliminate the free cancer cells present in the peritoneal cavity. LHIPEC is conducted with a usage of $4000 \mathrm{ml}$ sodium chloride solution combined with anticancer agents, heated up to $42-43^{\circ} \mathrm{C}$. Next, a port is installed into peritoneal cavity of the hypogastric region, which allows repeated intraperitoneal administration of chemotherapeutic agents. Two weeks after LHIPEC, the first out of three planned NIPS cycles is carried out through this port.

The principal CRS and HIPEC procedure is done 3-4 weeks after the last cycle of preoperative chemotherapy. During the surgery, EIPL is performed before and after the particular part of resection, in order to remove free cancer cells from opened and damaged blood and lymphatic vessels. Cytoreductive surgery consists of gastrectomy extended with splenectomy and/or cholecystectomy in combination with at least D2 lymphadenectomy. At the end of the procedure, HIPEC is accomplished to eliminate micro-metastases in the peritoneal space and surface, which could be left over after cytoreduction and peritoneal lavage.

Recently, the first report verifying the reduction of PCI score after NLHIPEC and NIPS has been published [52]. Herein, NLHIPEC plus NIPS is considered a safe and effective method of eradicating peritoneal free cancer cells before CRS. Additionally, the PCI score is reduced to less than the cut-off level by LHIPEC plus NIPS, resulting in an increased rate of complete cytoreduction and improvement in prognosis after CRS.

\section{CONCLUSIONS}

There is sufficient evidence that gastrectomy as a component of the CRS and HIPEC after preoperative systemic chemotherapy is a safe and feasible treatment option for patients with advanced GC and positive peritoneal cytology and/or limited PM. Although this is not yet standard treatment in Europe, eligible patients with locally advanced
(cT3-4) GC should be offered to enter clinical studies. They could be included if the primary GC is considered resectable, and positive peritoneal cytology and/or limited PM $(\mathrm{PCI}<12)$ are confirmed by diagnostic laparoscopy. In such patients, primary induction systemic chemotherapy should be administered without disease progression. Thereafter, a laparotomy should be conducted with intention to perform the CRS (complete removal of all macroscopically visible tumour deposits) by means of a (sub)total gastrectomy with at least D2 lymph node dissection. In patients with the PCI score above the cut-off level ( $>12)$, NLHIPEC plus NIPS may result in an increased rate of complete cytoreduction and improvement in prognosis after CRS. Still, further improvement of this multimodal strategy is needed in order to optimize the treatment of an otherwise incurable disease with dismal prognosis.

\section{REFERENCES}

1. Nakamura K, Ueyama T, Yao T, Xuan ZX, Ambe K, Adachi Y et al. Pathology and prognosis of gastric carcinoma. Findings in 10,000 patients who underwent primary gastrectomy. Cancer 1992, 70(5):1030-1037.

2. Gunderson LL, Sosin H. Adenocarcinoma of the stomach: areas of failure in a re-operation series (second or symptomatic look) clinicopathologic correlation and implications for adjuvant therapy. Int J Radiat Oncol Biol Phys 1982, 8(1):1-11.

3. Fujitani K, Yang HK, Mizusawa J, Kim YW, Terashima M, Han SU et al. Gastrectomy plus chemotherapy versus chemotherapy alone for advanced gastric cancer with a single non-curable factor (REGATTA): a phase 3, randomised controlled trial. Lancet Oncol 2016, 17(3):309-318.

4. Kerkar SP, Kemp CD, Duffy A, Kammula US, Schrump DS, Kwong $\mathrm{KF}$ et al. The GYMSSA trial: a prospective randomized trial comparing gastrectomy, metastasectomy plus systemic therapy versus systemic therapy alone. Trials 2009, 10:121.

5. Dai Q, Jiang L, Lin RJ, Wei KK, Gan LL, Deng CH et al. Adjuvant chemoradiotherapy versus chemotherapy for gastric cancer: a metaanalysis of randomized controlled trials. Journal of surgical oncology 2015, 111(3):277-284.

6. Desiderio J, Chao J, Melstrom L, Warner S, Tozzi F, Fong Y et al. The 30-year experience-A meta-analysis of randomised and highquality non-randomised studies of hyperthermic intraperitoneal chemotherapy in the treatment of gastric cancer. Eur J Cancer 2017, 79:1-14.

7. Conlon KC, Karpeh MS, Jr. Laparoscopy and laparoscopic ultrasound in the staging of gastric cancer. Semin Oncol 1996, 23(3):347-351.

8. Stell DA, Carter CR, Stewart I, Anderson JR. Prospective comparison of laparoscopy, ultrasonography and computed tomography in the staging of gastric cancer. Br J Surg 1996, 83(9):1260-1262.

9. Bryan RT, Cruickshank NR, Needham SJ, Moffitt DD, Young JA, Hallissey MT et al. Laparoscopic peritoneal lavage in staging gastric and oesophageal cancer. Eur J Surg Oncol 2001, 27(3):291-297.

10. Ribeiro U, Jr., Gama-Rodrigues JJ, Safatle-Ribeiro AV, Bitelman B, Ibrahim RE, Ferreira MB et al. Prognostic significance of intraperitoneal free cancer cells obtained by laparoscopic peritoneal lavage in patients with gastric cancer. Journal of gastrointestinal surgery : official journal of the Society for Surgery of the Alimentary Tract 1998, 2(3):244-249.

11. Bentrem D, Wilton A, Mazumdar M, Brennan M, Coit D. The value of peritoneal cytology as a preoperative predictor in patients with gastric carcinoma undergoing a curative resection. Annals of surgical oncology 2005, 12(5):347-353.

12. Coburn N, Seevaratnam R, Paszat L, Helyer L, Law C, Swallow $\mathrm{C}$ et al. Optimal management of gastric cancer: results from an international RAND/UCLA expert panel. Annals of surgery 2014, 259(1):102-108. 
13. Dixon M, Cardoso R, Tinmouth J, Helyer L, Law C, Swallow C et al. What studies are appropriate and necessary for staging gastric adenocarcinoma? Results of an international RAND/UCLA expert panel. Gastric cancer : official journal of the International Gastric Cancer Association and the Japanese Gastric Cancer Association 2014, 17(2):377-382.

14. Fujimoto S, Shrestha RD, Kokubun M, Ohta M, Takahashi M, Kobayashi K et al. Intraperitoneal hyperthermic perfusion combined with surgery effective for gastric cancer patients with peritoneal seeding. Annals of surgery 1988, 208(1):36-41.

15. Yonemura Y, Fujimura T, Fushida S, Takegawa S, Kamata T, Katayama K et al. Hyperthermo-chemotherapy combined with cytoreductive surgery for the treatment of gastric cancer with peritoneal dissemination. World J Surg 1991, 15(4):530-535; discussion 535-536.

16. Fujimoto S, Takahashi M, Mutou T, Kobayashi K, Toyosawa T, Isawa $\mathrm{E}$ et al. Improved mortality rate of gastric carcinoma patients with peritoneal carcinomatosis treated with intraperitoneal hyperthermic chemoperfusion combined with surgery. Cancer 1997, 79(5):884-891.

17. Yonemura Y, Kawamura T, Bandou E, Takahashi S, Sawa T, Matsuki $\mathrm{N}$. Treatment of peritoneal dissemination from gastric cancer by peritonectomy and chemohyperthermic peritoneal perfusion. Br J Surg 2005, 92(3):370-375.

18. Glehen O, Gilly FN, Arvieux C, Cotte E, Boutitie F, Mansvelt B et al. Peritoneal carcinomatosis from gastric cancer: a multi-institutional study of 159 patients treated by cytoreductive surgery combined with perioperative intraperitoneal chemotherapy. Annals of surgical oncology 2010, 17(9):2370-2377.

19. Yonemura Y, Bandou E, Kinoshita K, Kawamura T, Takahashi S, Endou $\mathrm{Y}$ et al. Effective therapy for peritoneal dissemination in gastric cancer. Surg Oncol Clin N Am 2003, 12(3):635-648.

20. Canbay E, Mizumoto A, Ichinose M, Ishibashi H, Sako S, Hirano M et al. Outcome data of patients with peritoneal carcinomatosis from gastric origin treated by a strategy of bidirectional chemotherapy prior to cytoreductive surgery and hyperthermic intraperitoneal chemotherapy in a single specialized center in Japan. Annals of surgical oncology 2014, 21(4):1147-1152.

21. Coccolini F, Cotte E, Glehen O, Lotti M, Poiasina E, Catena F et al. Intraperitoneal chemotherapy in advanced gastric cancer. Metaanalysis of randomized trials. Eur J Surg Oncol 2014, 40(1):12-26.

22. Brar SS, Mahar AL, Helyer LK, Swallow C, Law C, Paszat L et al. Processes of care in the multidisciplinary treatment of gastric cancer: results of a RAND/UCLA expert panel. JAMA surgery 2014, 149(1):18-25.

23. Yang X-J, Huang C-Q, Suo T, Mei L-J, Yang G-L, Cheng F-L et al. Cytoreductive Surgery and Hyperthermic Intraperitoneal Chemotherapy Improves Survival of Patients with Peritoneal Carcinomatosis from Gastric Cancer: Final Results of a Phase III Randomized Clinical Trial. Annals of surgical oncology 2011, 18(6):1575-1581.

24. Yonemura Y. A new bidirectional intraperitoneal and systemic induction chemotherapy (BISIC) for the peritoneal metastasis from gastric cancer in neoadjuvant setting. Integrative Cancer Science and Therapeutics 2014, 1(2):26-29.

25. Yonemura Y, Elnemr A, Endou Y, Ishibashi H, Mizumoto A, Miura $\mathrm{M}$ et al. Surgical results of patients with peritoneal carcinomatosis treated with cytoreductive surgery using a new technique named aqua dissection. Gastroenterol Res Pract 2012, 2012:521487.

26. Mizumoto A, Canbay E, Hirano M, Takao N, Matsuda T, Ichinose M et al. Morbidity and mortality outcomes of cytoreductive surgery and hyperthermic intraperitoneal chemotherapy at a single institution in Japan. Gastroenterol Res Pract 2012, 2012:836425.

27. Roviello F, Caruso S, Neri A, Marrelli D. Treatment and prevention of peritoneal carcinomatosis from gastric cancer by cytoreductive surgery and hyperthermic intraperitoneal chemotherapy: Overview and rationale. European Journal of Surgical Oncology (EJSO) 2013, 39(12):1309-1316.

28. Glehen O, Passot G, Villeneuve L, Vaudoyer D, Bin-Dorel S, Boschetti G et al. GASTRICHIP: D2 resection and hyperthermic intraperitoneal chemotherapy in locally advanced gastric carcinoma: a randomized and multicenter phase III study. BMC Cancer 2014, 14(1):183.
29. Rau B, Loeffler M, Rau H-G, Sulkowski U, Kuhlmann J, Weimann A, Keck T, Angele M, Topp SA, Koenigsrainer A et al: Perioperative chemotherapy and cytoreductive surgery with versus without HIPEC in gastric cancer with limited peritoneal metastases: A randomized phase III study (GASTRIPEC). Journal of Clinical Oncology 2015, 33(15_suppl):TPS4132-TPS4132.

30. Coccolini F, Catena F, Glehen O, Yonemura Y, Sugarbaker PH, Piso $\mathrm{P}$ et al. Complete versus incomplete cytoreduction in peritoneal carcinosis from gastric cancer, with consideration to PCI cut-off. Systematic review and meta-analysis. European Journal of Surgical Oncology (EJSO) 2015, 41(7):911-919.

31. Boerner T, Graichen A, Jeiter T, Zemann F, Renner P, Marz L et al. CRS-HIPEC Prolongs Survival but is Not Curative for Patients with Peritoneal Carcinomatosis of Gastric Cancer. Annals of surgical oncology 2016, 23(12):3972-3977.

32. Esquivel J. Technology of hyperthermic intraperitoneal chemotherapy in the United States, Europe, China, Japan, and Korea. Cancer journal (Sudbury, Mass) 2009, 15(3):249-254.

33. van der Kaaij RT, Braam HJ, Boot H, Los M, Cats A, Grootscholten $\mathrm{C}$ et al. Treatment of Peritoneal Dissemination in Stomach Cancer Patients With Cytoreductive Surgery and Hyperthermic Intraperitoneal Chemotherapy (HIPEC): Rationale and Design of the PERISCOPE Study. JMIR Res Protoc 2017, 6(7):e136.

34. Koh J-L, Yan TD, Glenn D, Morris DL. Evaluation of Preoperative Computed Tomography in Estimating Peritoneal Cancer Index in Colorectal Peritoneal Carcinomatosis. Annals of surgical oncology 2009, 16(2):327-333.

35. Pfannenberg C, Konigsrainer I, Aschoff P, Oksuz MO, Zieker D, Beckert $S$ et al. (18)F-FDG-PET/CT to select patients with peritoneal carcinomatosis for cytoreductive surgery and hyperthermic intraperitoneal chemotherapy. Annals of surgical oncology 2009, 16(5):1295-1303.

36. Hong SH, Shin YR, Roh SY, Jeon EK, Song KY, Park CH et al. Treatment outcomes of systemic chemotherapy for peritoneal carcinomatosis arising from gastric cancer with no measurable disease: retrospective analysis from a single center. Gastric cancer : official journal of the International Gastric Cancer Association and the Japanese Gastric Cancer Association 2013, 16(3):290-300.

37. Valle M, Federici O, Garofalo A. Patient selection for cytoreductive surgery and hyperthermic intraperitoneal chemotherapy, and role of laparoscopy in diagnosis, staging, and treatment. Surg Oncol Clin N Am 2012, 21(4):515-531.

38. Nath J, Moorthy K, Taniere P, Hallissey M, Alderson D. Peritoneal lavage cytology in patients with oesophagogastric adenocarcinoma. Br J Surg 2008, 95(6):721-726.

39. Lee SD, Ryu KW, Eom BW, Lee JH, Kook MC, Kim YW. Prognostic significance of peritoneal washing cytology in patients with gastric cancer. Br J Surg 2012, 99(3):397-403.

40. Lisiecki R, Spychala A, Pater K, Murawa D. Analysis Of Risk Factors Of Positive Peritoneal Cytology In Patients Treated For Gastric Cancer-Preliminary Report. Polski przeglad chirurgiczny 2015, 87(10):506-512.

41. Bando E, Yonemura Y, Takeshita Y, Taniguchi K, Yasui T, Yoshimitsu $\mathrm{Y}$ et al. Intraoperative lavage for cytological examination in 1,297 patients with gastric carcinoma. Am J Surg 1999, 178(3):256-262.

42. Ikeguchi M, Oka A, Tsujitani S, Maeta M, Kaibara N. Relationship between area of serosal invasion and intraperitoneal free cancer cells in patients with gastric cancer. Anticancer Res 1994, 14(5B):2131-2134

43. Fujiwara Y, Doki Y, Taniguchi H, Sohma I, Takiguchi S, Miyata H et al. Genetic detection of free cancer cells in the peritoneal cavity of the patient with gastric cancer: present status and future perspectives. Gastric cancer : official journal of the International Gastric Cancer Association and the Japanese Gastric Cancer Association 2007, 10(4):197-204.

44. Huang JY, Xu YY, Sun Z, Zhu Z, Song YX, Guo PT et al. Comparison different methods of intraoperative and intraperitoneal chemotherapy for patients with gastric cancer: a meta-analysis. Asian Pac J Cancer Prev 2012, 13(9):4379-4385. 
45. Sun J, Song Y, Wang Z, Gao P, Chen X, Xu Y et al. Benefits of hyperthermic intraperitoneal chemotherapy for patients with serosal invasion in gastric cancer: a meta-analysis of the randomized controlled trials. BMC Cancer 2012, 12:526.

46. Mi DH, Li Z, Yang KH, Cao N, Lethaby A, Tian JH et al. Surgery combined with intraoperative hyperthermic intraperitoneal chemotherapy (IHIC) for gastric cancer: a systematic review and meta-analysis of randomised controlled trials. Int J Hyperthermia 2013, 29(2):156-167.

47. Yan TD, Black D, Sugarbaker PH, Zhu J, Yonemura Y, Petrou G et al. A Systematic Review and Meta-analysis of the Randomized Controlled Trials on Adjuvant Intraperitoneal Chemotherapy for Resectable Gastric Cancer. Annals of surgical oncology 2007, 14(10):2702-2713.

48. Braam HJ, Schellens JH, Boot H, van Sandick JW, Knibbe CA, Boerma D et al. Selection of chemotherapy for hyperthermic intraperitoneal use in gastric cancer. Critical Reviews in Oncology/ Hematology 2015, 95(3):282-296.
49. Scaringi S, Kianmanesh R, Sabate JM, Facchiano E, Jouet P, Coffin $\mathrm{B}$ et al. Advanced gastric cancer with or without peritoneal carcinomatosis treated with hyperthermic intraperitoneal chemotherapy: a single western center experience. Eur J Surg Oncol 2008, 34(11):1246-1252.

50. Coccolini F, Celotti A, Ceresoli M, Montori G, Marini M, Catena F et al. Hyperthermic intraperitoneal chemotherapy (HIPEC) and neoadjuvant chemotherapy as prophylaxis of peritoneal carcinosis from advanced gastric cancer-effects on overall and disease free survival. J Gastrointest Oncol 2016, 7(4):523-529.

51. Yonemura Y, Canbay E, Li Y, Coccolini F, Glehen O, Sugarbaker PH et al. A comprehensive treatment for peritoneal metastases from gastric cancer with curative intent. European Journal of Surgical Oncology (EJSO) 2016, 42(8):1123-1131.

52. Yonemura Y, Ishibashi H, Hirano M, Mizumoto A, Takeshita K, Noguchi K et al. Effects of Neoadjuvant Laparoscopic Hyperthermic Intraperitoneal Chemotherapy and Neoadjuvant Intraperitoneal/ Systemic Chemotherapy on Peritoneal Metastases from Gastric Cancer. Annals of surgical oncology 2017, 24(2):478-485. 